\title{
Terahertz Long-Distance Propagation Loss in the Air
}

This paper was downloaded from TechRxiv (https://www.techrxiv.org).

\section{LICENSE}

CC BY 4.0

SUBMISSION DATE / POSTED DATE

$15-01-2022$ / 20-01-2022

CITATION

guo, yingjiang; Xu, Kai-Da; Deng, Xianjin (2022): Terahertz Long-Distance Propagation Loss in the Air. TechRxiv. Preprint. https://doi.org/10.36227/techrxiv.18480425.v1

$\mathrm{DOI}$

10.36227/techrxiv.18480425.v1 


\title{
Terahertz Long-Distance Propagation Loss in the Air
}

\author{
Ying-Jiang Guo, Member, IEEE, Kai-Da Xu, Senior Member, IEEE, and Xianjin Deng
}

\begin{abstract}
Terahertz communications demonstrate advantages of wide bandwidth and low latency compared to the microwave counterparts but suffer from high propagation loss in the air. Little research focuses on long-distance (i.e., distance $>1 \mathrm{~km}$ ) terahertz propagation characteristics in the air, posing challenges on long-distance terahertz wireless applications. In this paper, the terahertz long-distance propagation characteristics are explored and studied with the help of the measurement system operating at 220 GHz. Based on the measurement systems, we conduct out-door experiments with a propagation distance of $2.5 \mathrm{~km}$ to obtain the terahertz propagation loss in the air. Additionally, a comparison between the measured propagation loss and the calculated one based on models issued by the international telecommunication union (ITU) shows an intrinsic loss gap between these two results, which is found and explained for the first time in the above-100 $\mathrm{GHz}$ long-distance communication applications. With the analysis of the collected data, we propose a gap-compensation method to obtain a more accurate prediction for the propagation loss in the air. With the help of the proposed systems and corresponding measurements, we could give insight into the terahertz propagation and pave the way for future terahertz long-distance applications.
\end{abstract}

Index Terms-Long distance, out-door experiments, propagation loss, path loss, propagation measurement, terahertz

\section{INTRODUCTION}

$\mathrm{T}$ ERAHERTZ communications have attracted many interests in both industrial and academic communities due to the benefits of wide bandwidth and low latency. However, it also suffers from high propagation loss in the air, which increases the difficulties of developing the terahertz wireless applications, especially for long-distance terahertz communication [1-3]. To solve this challenge, many scientists and researchers made various efforts (such as enhancing the output power of the devices, building high-gain antennas, optimization the management of the wireless channels [4-10]). Among these works, the wireless channel model plays a critical role in designing the terahertz wireless systems [11-16]. Because only with explicit knowledge of the terahertz wireless channel could we assign the design requirements to every part

Y. J. Guo and X. Deng are with the Microsystem and Terahertz Research Center, China Academy of Engineering Physics, Chengdu, China. (e-mail: guoyingjiang1@gmail.com)

K.-D. $\mathrm{Xu}$ is with the School of Information and Communications Engineering, Xi'an Jiaotong University, Xi'an 710049, China. (e-mail: kaidaxu@ieee.org) of the system, including components, antennas, circuits, and materials.

Much research was carried out on wireless channel models at millimeter-wave and terahertz bands. Especially in the sub-100 $\mathrm{GHz}$ community, many research staffs published piles of communication-channel reports and surveys $[17,18]$, while in the above-100 GHz community, fewer works related to channels were reported. To promote the development of the above-100 GHz wireless applications, many research institutes conduct above-100 GHz in-door and out-door studies to study terahertz wireless communications. In terms of the out-door scenario, the influence of the atmospheric composition and the weather condition, including snow, rain, fog, and typhoon, was studied, and the corresponding equations were also derived for better prediction of the path loss [19-28]. Meanwhile, various in-door channel-modeling methods and measurement solutions were developed [29-32].

Most of these above-100 $\mathrm{GHz}$ works focus on in-door propagation measurements or the short-distance (i.e., distance $<600 \mathrm{~m}$ ) out-door experiments, which could not provide insight into the terahertz long-distance propagation characteristics. In the short-distance case, the differences between the measured and calculated air loss are minor and easy to be neglected, while these differences would be more evident for the long-distance case. Some standardization organizations have released models and methods for terahertz communication channels [33-36]. Also, people developed more theories and formulas for better prediction of path loss [37-40]. However, for the above- $100 \mathrm{GHz}$ scenario, these models could not be well applied to predict the terahertz long-distance propagation loss, because they are constructed through the theoretical extension from low-frequency models without experimental validation.

In order to tackle these challenges mentioned above, in this paper, we propose a propagation measurement system operating at $220 \mathrm{GHz}$ to measure the terahertz long-distance propagation loss in the air. Detailed design information of the proposed measurement system is illustrated in Section II. The out-door experiments at $220 \mathrm{GHz}$ with a distance of $2.5 \mathrm{~km}$ are performed independently in Mianyang, China, as demonstrated in Section III. Note that for the $220 \mathrm{GHz}$ experiments, the measured propagation loss is more significant than the predicted loss calculated by international telecommunication union (ITU) models. It indicates that the existing models issued by ITU are not applicable for terahertz propagation-loss prediction. In Section IV, the measured data is carefully 
analyzed and discussed to explore the possible reasons for such a loss gap, and the suggestions of the modification on the existing ITU models are also provided. Finally, Section V concludes the paper.

\section{MEASUREMENT SySTEMS}

Fig. 1 is the architecture view of the proposed measurement system operating at $220 \mathrm{GHz}$, which consists of terahertz front ends, intermediate frequency (IF)/digital signal processing platform, and the post-processing methods developed on the PC. The IF/digital signal processing platform has two channels, enabling the simultaneous processing of two-channel input IF signals. In the measurement scenario, the transmitter (TX) and the receiver $(\mathrm{RX})$ parts of the measurement systems are placed independently at different locations, and we measure the line-of-sight propagation loss by observing the power of the IF signal. The line-of-sight propagation loss is the sum of free-space path loss and air loss. The former loss would be constant once the propagation distance is fixed. Nevertheless, the latter loss (i.e., air loss) was influenced by weather factors and the composition of air. The total propagation loss in the air equals the combination of these two losses. In this work, we mainly focus on the prediction and measurement of the air loss.

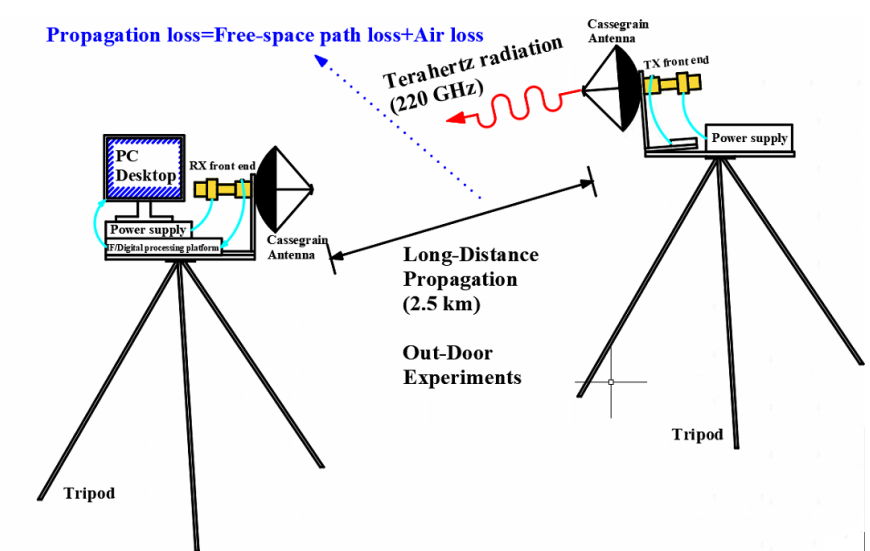

Fig. 1 Architecture view and measurement scenario of the proposed measurement systems.

As illustrated in Fig. 2, the front end of the proposed system consists of three parts: the antennas, the TX, and the RX. The TX transmits the terahertz signal, while the RX receives it and converts it to an IF signal. We deploy the Cassegrain antennas to bridge the terahertz wave and current. The $220 \mathrm{GHz}$ TX part completes the frequency-conversion work by the $\times 8$ frequency multiplier and the mixer, while the solid-state power amplifier enhances the signal's power. In the $220 \mathrm{GHz}$ RX part, the signal from the air is efficiently collected by the antenna and then amplified by the low-noise amplifier. Two cascaded mixers carry the down-conversion task out, where we apply two corresponding local oscillators to the mixers to obtain the final IF signal of $10 \mathrm{MHz}$.

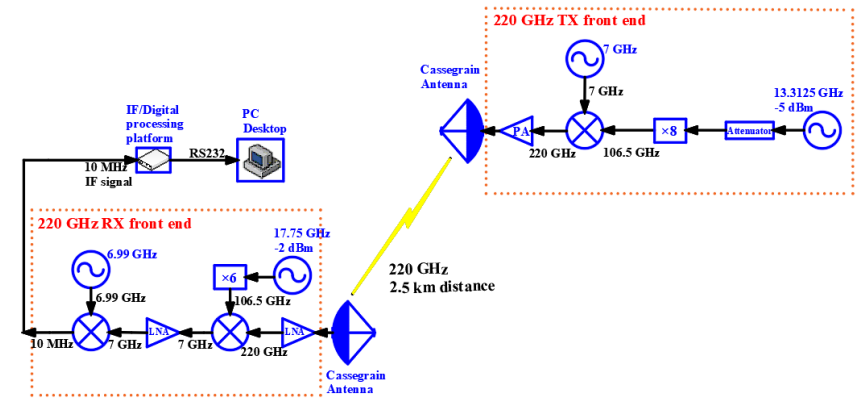

Fig. 2 Schematic view of the front ends.

Because the output power of the front end would decrease as the propagation distance increases, the IF/digital platform would be out of work if the input power for such platform is lower than the baseline. On the other hand, once the input power for the platform exceeds the maximum limit, it would harm the measurement accuracy. Therefore, the TX, RX, and antennas are carefully designed with an adequate link budget to obtain appropriate power for the link. Table I is the link budget for the proposed measurement system.

In the link-budget calculation, we assume the air loss at 220 $\mathrm{GHz}$ is $25 \mathrm{~dB}$, and set the distance the same as that in practical measurement. In Table I, the free-space path loss $L_{\text {free }}$ denotes the loss which is only caused by the electromagnetic diffusion loss in the free space, while the air loss $L_{\mathrm{a}}$ is the loss which is only due to the air or small particles in the air, including the snowflake, raindrop, and dust.

Since the relationship of these parameters in Table I can be calculated by equation (1), the known parameters to such equation can be substituted to derive the desired parameter (e.g., power of IF signal: $\left.P_{\mathrm{IF}}\right)$. In other words, once the air loss $\left(L_{\mathrm{a}}\right)$ could be predicted accurately, for the long-distance propagation case, it would be easy to meet the design requirement by assigning appropriate parameters (i.e., $G_{\mathrm{atx}}, G_{\mathrm{arx}}, G_{\mathrm{rx}}, P_{\mathrm{t}}$ ) to each part of the front ends.

$$
P_{\mathrm{IF}}=P_{\mathrm{t}}+G_{\text {atx }}-L_{\text {free }}-L_{\mathrm{a}}+G_{\mathrm{arx}}+G_{\mathrm{rx}}-L_{\mathrm{m}}
$$

TABLE I

LINK BUDGET FOR $220 \mathrm{GHz}$ FRONT END

\begin{tabular}{||c|c|c||}
\hline Main parameters & UNIT & Values \\
\hline Transmitting power: $P_{t}$ & $\mathrm{dBm}$ & -3.6 \\
\hline Gain of TX antenna: $G_{a t x}$ & $\mathrm{dBi}$ & 50 \\
\hline Distance between TX and RX & $\mathrm{km}$ & 2.5 \\
\hline Free-space path loss: $L_{f r e e}$ & $\mathrm{~dB}$ & 147.24 \\
\hline Estimated air loss (RH90\%): $L_{a}$ & $\mathrm{~dB}$ & 25 \\
\hline Gain of RX antenna: $G_{a r x}$ & $\mathrm{dBi}$ & 50 \\
\hline Gain of RX front end: $G_{r x}$ & $\mathrm{~dB}$ & 70 \\
\hline $\begin{array}{c}\text { Loss due to the misalignment of } \\
\text { the antennas: } L_{m}\end{array}$ & $\mathrm{~dB}$ & 0 \\
\hline Power of IF signal: $P_{I F}$ & $\mathrm{dBm}$ & -5.78 \\
\hline
\end{tabular}

In addition to the front ends, the proposed measurement system consists of the IF/digital signal processing platform and the PC-based post-algorithm platform. Fig. 3 is the schematic view of the platform. We built the IF/Digital signal processing platform by an FPGA chip and an analog to digital converter (ADC) with two independent input channels. The FPGA named "XC7K325T-2FFG676I" could control the ADC as desired to 
complete the task of analog to digital conversion. Meanwhile, the FPGA is also utilized to realize the data communication between the platform and the PC. In order to tackle the unforeseen and unknown challenges, the FPGA in this platform has many multiplexing ports and hardware accelerators, which are reserved for future extended development. Besides, the ADCs named "ADS42LB69" support simultaneous two-channel sampling, each with a maximum sampling rate of 250 MSPS. Since the sampling rate of ADC is 25 times larger than the frequency of the IF (i.e., $10 \mathrm{MHz}$ ), the analog IF signal could be converted to a digital signal completely and nearly without defects. The raw data converted by the ADC would be temporarily stored in the FPGA chip and then transferred to the $\mathrm{PC}$ through the communication connection.

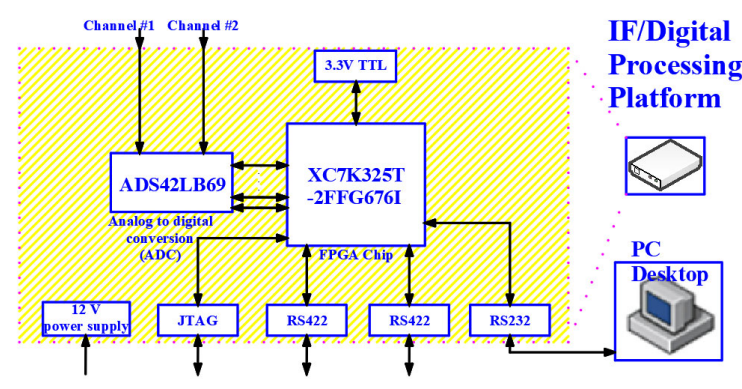

Fig. 3 IF/digital signal processing platform.

We developed a post-processing method based on PC to process and store the raw data to extract the power information. The flow chart of the proposed processing method is illustrated in Fig. 4. Firstly, the raw data is collected and converted to a digital signal by the platform and then is processed with the FFT conversion. Based on the spectrum results of the FFT conversion, we perform a peak-value search to obtain the position and value of the spectrum peak. Before the peak-value search, a digital bandpass filter suppresses the unwanted signal outside of the desired frequency range. In this project, the operation range of the bandpass filter is selected as 9-11 MHz. Then, the specified number (i.e., $n$ ) of the data points around the peak is added to obtain the $k$ value. We could look up the under-test IF-signal power based on the calculated value of $k$, based on the calibration database. Such calibration database is created before deploying such processing platform and methods.

The power measurement accuracy of the proposed processing method and the corresponding IF/digital processing platform is validated through experiments. Fig. 5 is the set-up of the measurements. We feed the IF signal with desired power and signal to noise ratio (SNR) to the processing platform, read the power value through the proposed method in the PC, and compare these two results (i.e., reading value in the PC and the setting value of IF signal). Signal generator and noise generator are applied to mimic the power and SNR of the IF signal as desired. The comparison between the set values and the measured results is illustrated in Table II, where the SNR of the under-test IF signal is $15 \mathrm{~dB}$. It could be found that the proposed platform and method could achieve an accurate power value of IF signal with errors smaller than $1 \mathrm{~dB}$ when the power ranges from $-30 \mathrm{dBm}$ to $0 \mathrm{dBm}$. Besides, it is found that the low SNR would harm power measurement accuracy. As the SNR decreases smaller than $10 \mathrm{~dB}$, the power measurement error would reach higher than $1 \mathrm{~dB}$ when the power ranges from $-30 \mathrm{dBm}$ to $0 \mathrm{dBm}$, implying that the IF signal with appropriate power and SNR is necessary for the processing platform and method to obtain an accurate measurement of the IF signal. We carefully design the TX and RX parts to assure that the power of the IF signal locates in the range of $-30 \sim 0 \mathrm{dBm}$, and the SNR is higher than $15 \mathrm{~dB}$. Note that the errors in Table II are the average difference between the reading value of the spectrum analyzer and the repeated measured results (i.e., results $\# 1$ to \#4). Except that the unit of "Errors" is dB, the unit of the other values in Table II is $\mathrm{dBm}$. The values of "Signal Generator" and "Spectrum Analyzer" denote the nominal values set by the signal generator and measured by the spectrum analyzer, respectively.

TABLE II

COMPARISON BETWEEN THE SETTING VALUE OF SIGNAL GENERATOR AND THE MEASURED RESULTS FOR EVALUATION OF THE POWER-TEST ACCURACY

\begin{tabular}{||c|c|c|c|c|c|c||}
\hline $\begin{array}{c}\text { Signal } \\
\text { Generator }\end{array}$ & $\begin{array}{c}\text { Spectrum } \\
\text { Analyzer }\end{array}$ & $\begin{array}{c}\text { Results } \\
\# \mathbf{1}\end{array}$ & $\begin{array}{c}\text { Results } \\
\mathbf{\# 2}\end{array}$ & $\begin{array}{c}\text { Results } \\
\mathbf{3}\end{array}$ & $\begin{array}{c}\text { Results } \\
\mathbf{\# 4}\end{array}$ & Errors \\
\hline 0 & -0.65 & 0.25 & 0.28 & 0.19 & 0.23 & 0.8875 \\
\hline-7.5 & -7.54 & -6.55 & -6.6 & -6.52 & -6.49 & 0.7675 \\
\hline-15 & -15.52 & -14.58 & -14.6 & -14.53 & -14.49 & 0.64 \\
\hline-22 & -22.54 & -22.06 & -22.15 & -22.18 & -22.29 & 0.37 \\
\hline-26 & -26.16 & -25.24 & -25.46 & -25.37 & -25.38 & 0.7975 \\
\hline-30 & -30.08 & -27.78 & -27.82 & -27.94 & -27.9 & 2.22 \\
\hline-33 & -33.17 & -29.82 & -29.8 & -29.87 & -29.8 & 3.3475 \\
\hline
\end{tabular}

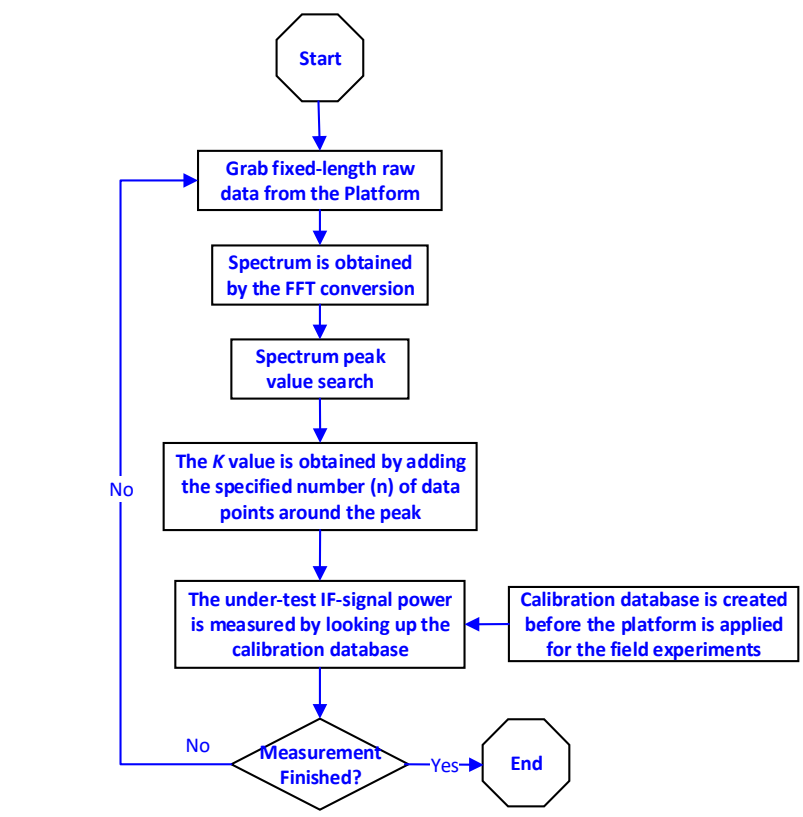

Fig. 4 Flow chart of the proposed post-processing method. 


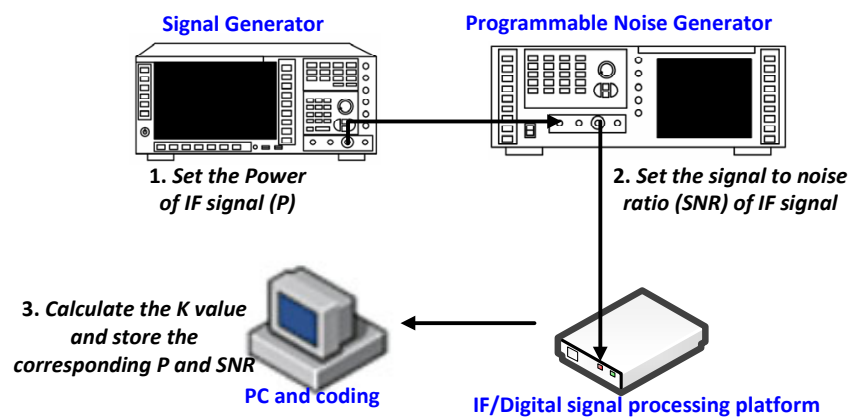

Fig. 5 Setup of the measurement to evaluate the power-test accuracy of the processing platform and method.

Before moving to the out-door experiments, the proposed systems, including the antennas, circuits, and components, are all tested carefully to confirm the performance, where all the parameters in Tables I and II were tested. Fig. 6 is the photo of fabricated systems under measurement.
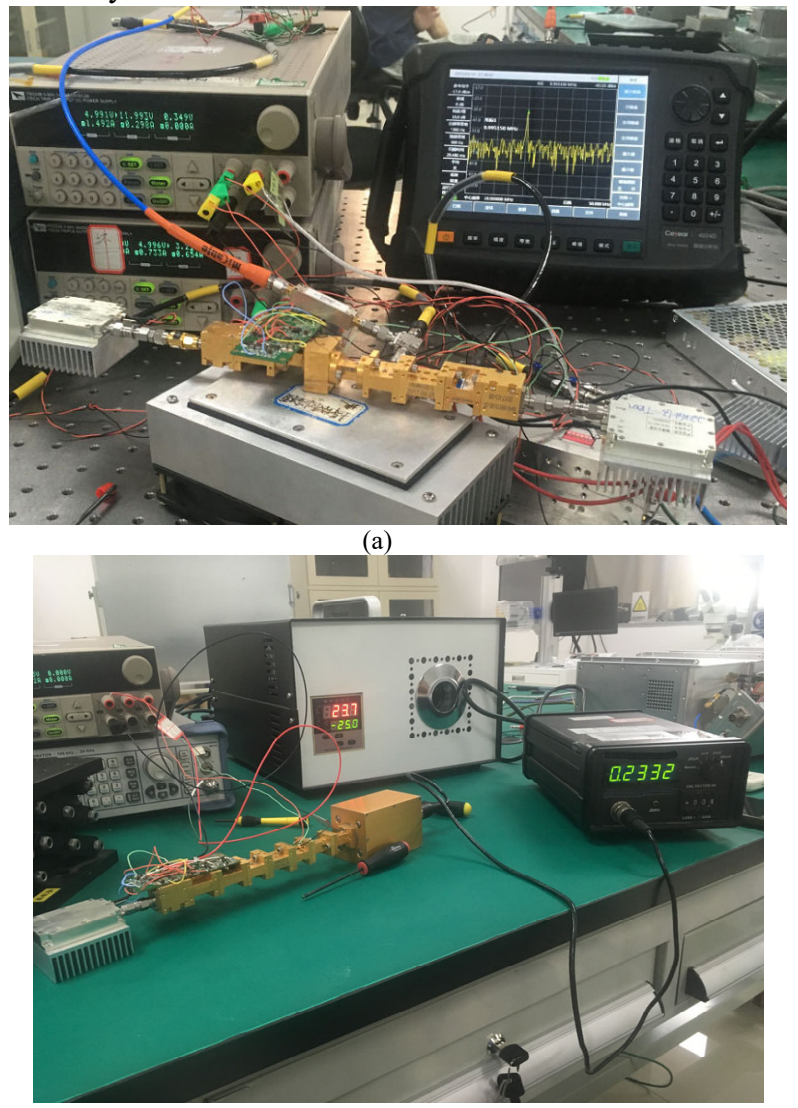

(b)

Fig. 6 Photo of the fabricated systems under measurement: (a) gain of RX front end; (b) transmitting power of TX front end.

To confirm the stability of the proposed systems, we conduct the measurement with the variance of temperature. The IF signal power is observed in this measurement to evaluate the stability with the variance of the environment. In the measurement, the TX and RX parts are directly connected by flange rectangular waveguide (hollow size: $1.0922 \times 0.5461$ $\mathrm{mm}^{2}$ ). In other words, such an experiment does not have antennas in the communication link. It is found that the power of IF signals in the $220 \mathrm{GHz}$ system shows minor changes (i.e., less than $1 \mathrm{~dB}$ ) as the temperature varies from -10 to $20^{\circ} \mathrm{C}$. Thus, these minor fluctuations $(<1 \mathrm{~dB})$ due to the temperature variation have few effects in accurately measuring propagation loss. Besides, to avoid the negative influence of the environment on the performance of the proposed terahertz front ends, the fans and heat-conduction metal plates are utilized to avoid the operation of systems in a high-temperature state.

\section{EXPERIMENTS AND RESULTS}

With the proposed measurement systems, we conduct out-door experiments to measure the propagation loss in the air. The TX and RX are placed separately in different locations, and the propagation loss is derived based on parameters in Tables I and II and the equation (1).

For the $220 \mathrm{GHz}$ measurement, the out-door experiment was carried out in Mianyang, Sichuan, China. The indication of TX/RX locations is demonstrated in Fig. 7(a). And Fig. 7(b) is the TX and RX front-end images in the out-door experiments. The distance between TX and RX is $2.5 \mathrm{~km}$. It is important to note that there are two antennas deployed in the $220 \mathrm{GHz}-\mathrm{RX}$ picture, where the antenna with smaller radius is for $220 \mathrm{GHz}$, while the other antenna is for $140 \mathrm{GHz}$. The radius of the 220 $\mathrm{GHz}$ antennas is $110 \mathrm{~m}$. The transmitting or receiving front ends are connected with the antennas by flange WR-4 rectangular waveguide (hollow size: $1.092 \times 0.546 \mathrm{~mm}^{2}$ ).

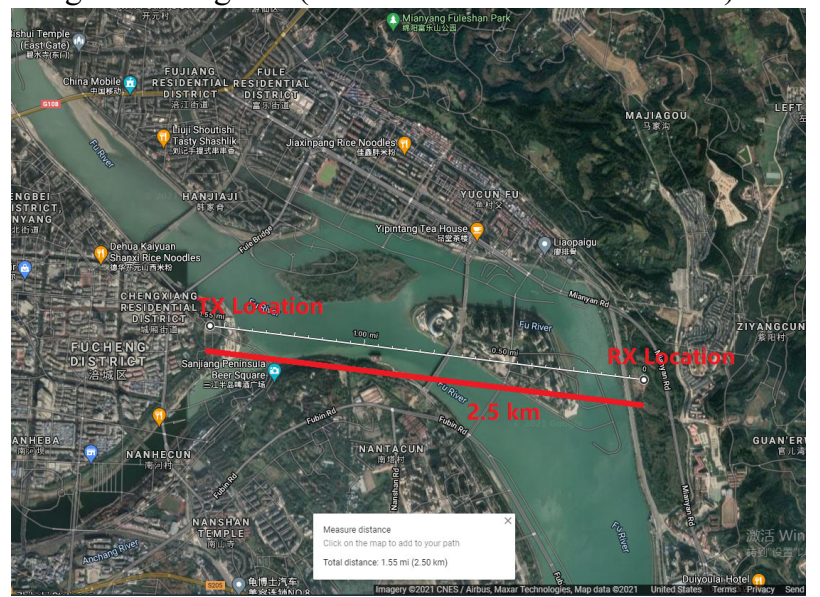

(a)
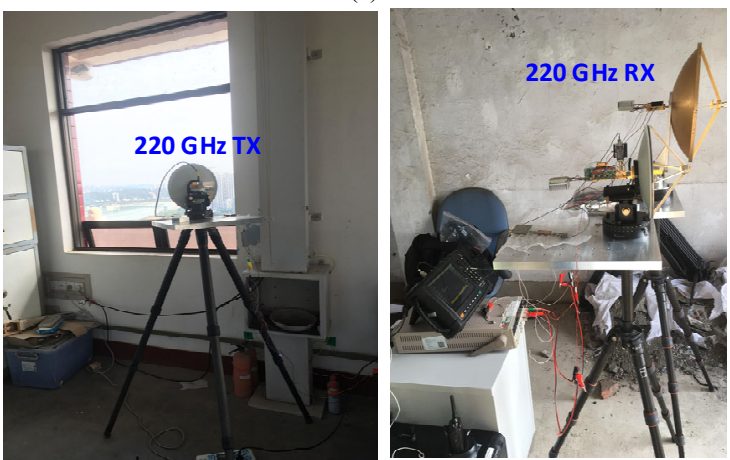

(b)

Fig. $7220 \mathrm{GHz}$ experiment: (a) indication of TX and RX locations, (b) images of the TX and RX in the out-door experiments.

Before measurement, the TX/RX antennas' directions are 
aligned to maintain the main radiation beams of TX and RX antennas on the same line. Once such a condition is satisfied, the power of the IF signal would achieve the maximum peak. Note that the link budget calculation in tables I and II is based on the assumption that the primary radiation beams of TX and $\mathrm{RX}$ antennas are aligned on the same line. Otherwise, the power of the IF signal would decrease, leading to errors for the propagation loss measurement. In order to complete the alignment process, the TX/RX front ends and antennas are fixed in the rotary table, which is used to tune the azimuth angle and pitch angle with acceptable resolution.

Fig. 8 compares the calculated air loss based on the ITU models and the measured air loss for the $220 \mathrm{GHz}$ experiments. Software coding is used to build the ITU models for path-loss prediction according to the ITU-released documents (i.e., "ITU-R P.676" and "ITU-R P.835") [34, 41], as illustrated in Fig. 9. The document "ITU-R P.676" indicates the attenuation by atmospheric gases and related effects, while the document of "ITU-R P.835" is the reference standard atmospheres. In the ITU-loss calculation process, the weather data is necessary and is collected through the China National Meteorological Administration.

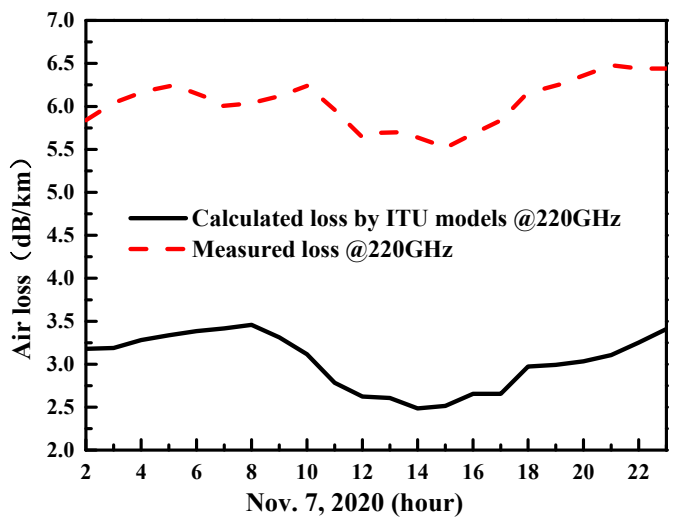

(a)

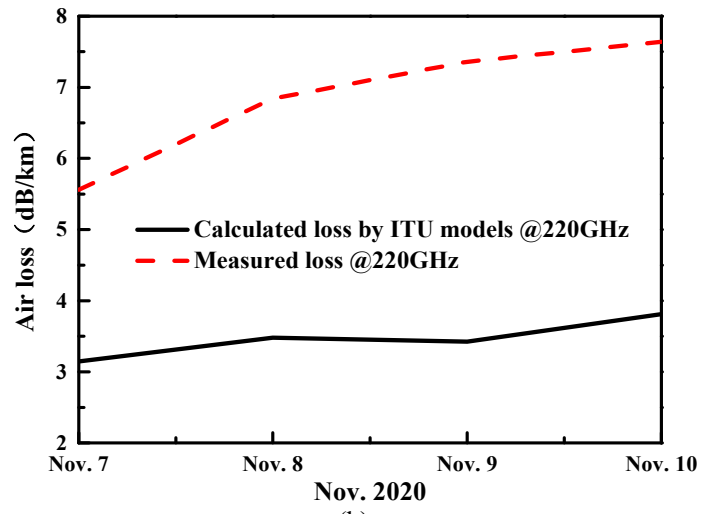

(b)

Fig. 8 Air loss comparison between the measured loss and the calculated loss by the ITU models for the $220 \mathrm{GHz}$ experiment: (a) single-day case on Nov. 07, 2020; (b) multi-days case from Nov. 07 to Nov. 10, 2020.

Fig. 8(a) is the measured air loss $(\mathrm{dB} / \mathrm{km})$ on Nov. 7, 2020, from 2:00 to 23:00, and Fig. 8(b) is the measured air loss from Nov. 7 to Nov. 10 in 2020. We observed a noticeable loss gap between the measured and calculated losses, and the measured results are more significant than the calculated ones. In the short-distance $(<1 \mathrm{~km})$ propagation case, the loss gap would shrink to smaller than $3 \mathrm{~dB}$. However, the situation is worse for the $2.5 \mathrm{~km}$ or even longer-distance propagation case with more significant propagation loss. We could derive the actual air loss by multiplying the propagation distance of $2.5 \mathrm{~km}$ by the loss data in Fig. 8. For the $2.5 \mathrm{~km}$ experiments, the loss gap is more than $6 \mathrm{~dB}$ (i.e., $>4$ times). The significance of the proposed long-distance experiment is to confirm the loss gap that is difficult to find in the short-distance measurements. With explicit knowledge of the terahertz long-distance wireless propagation, we could promote the development and deployment of the long-distance terahertz wireless technologies and applications.

The experimental results imply that the terahertz wireless system is more sensitive to air composition and weather conditions than its traditional microwave counterparts. We need enhanced gains/power for the terahertz front end and develop algorithms to tackle the challenge of loss fluctuation issues. Notably, the experiments clearly show that the existing ITU models cannot accurately predict the propagation loss in above-100 GHz bands.

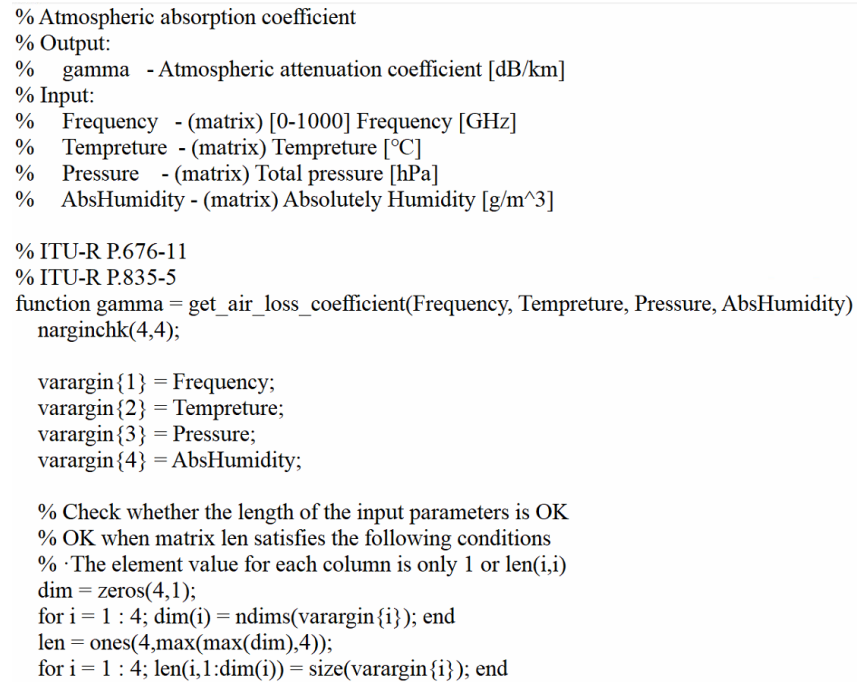

Fig. 9 Screenshot of the software coding for the ITU model.

\section{ANALYSIS AND Discussions}

In order to obtain a more accurate prediction of the propagation loss (i.e., air loss) for the $220 \mathrm{GHz}$ communications, we carry out the linear regression analysis to explore the relationship between the weather condition and the loss gap. The weather conditions (temperature, relative humidity, and wind speed) are analyzed. The software Excel is utilized to perform this analysis. It is found that for the 220 $\mathrm{GHz}$ propagation, the loss gap shows a strong relationship with the temperature and relative humidity (i.e., P-value $<0.01$ ). Fig. 10 is the screenshot of the linear regression analysis results between the loss gap and the temperature. 


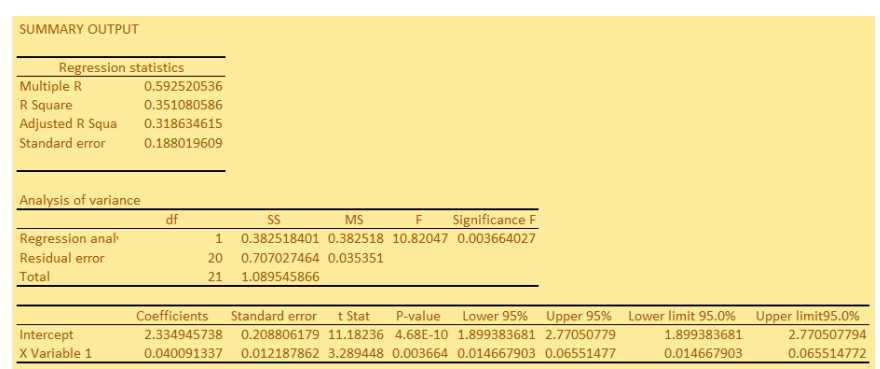

Fig. 10 Screenshot of the linear regression analysis results between the loss gap ( $y$ value) and the temperature ( $x$ value) for $220 \mathrm{GHz}$ propagation experiment

Based on these analysis results, we compensate the loss gap between the measured and calculated ones providing more accurate predictions. The software coding is utilized for the 220 $\mathrm{GHz}$ system to fit the raw data into a linear equation, as shown in equation (2).

$$
L_{\mathrm{a}}(220 \mathrm{GHz})=L_{\mathrm{ITU}}+k_{1} \times \text { Temp }+k_{2} \times \mathrm{Humi}+k_{3}
$$

where the $L_{\mathrm{a}}$ and $L_{\mathrm{ITU}}$ denote the calibrated air loss and the calculated air loss based on ITU models, respectively. The Temp and Humi represent the temperature and relative humidity. In order to fit the measured data to equation (2), $k_{1}, k_{2}$ and $k_{3}$ are optimized with the final value of -3.72627205191245 , -4.70733252541092 , and 158.132557461153

Fig. 11 compares the air loss $(\mathrm{dB} / \mathrm{km})$ between the calibrated results based on equation (2) and the measured results. With the help of the proposed equations, the loss gap between the measured results and the predicted ones is successfully decreased, facilitating the development and deployment of the long-distance terahertz wireless applications.

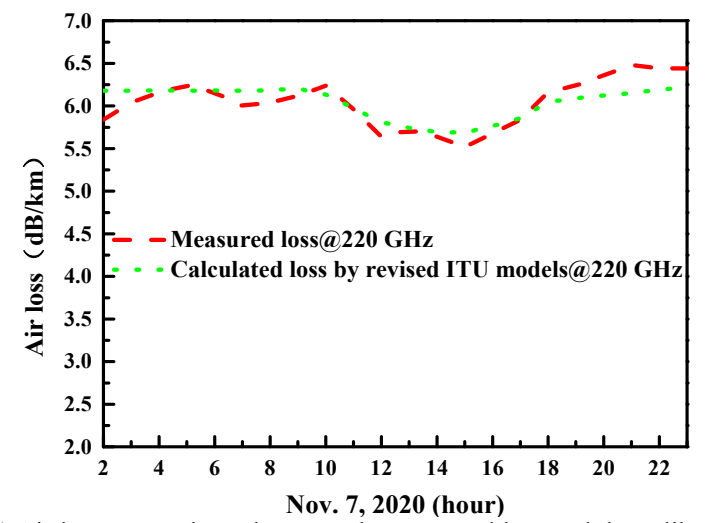

Fig. 11 Air loss comparisons between the measured loss and the calibrated air loss by the revised ITU models for the $220 \mathrm{GHz}$ experiments with the variance of hours.

Although equation (2) has been proposed to obtain more accurate loss-prediction results, we still did not find the physical reason behind the loss gap. In the future step of our work, to give insight into the loss gap, we will promote the exploration and study of the physical operation mechanism which leads to the loss gap. Moreover, based on the discovery of the physics reasons, the optimized loss prediction solutions would be proposed to obtain a more accurate prediction for the propagation loss in the long-distance terahertz wireless applications.

\section{CONCLUSION}

For the first time, the long-distance $(>1 \mathrm{~km})$ terahertz propagation loss is measured by the out-door experiments with the help of the proposed measurement systems. It is found that there is an intrinsic loss gap between the measured air loss and the calculated results based on ITU models. The relationship between the loss gap and the weather condition is analyzed, and the revision suggestions are presented based on regression analysis of the collected data. With the proposed compensation methods, the loss gap or the prediction error is shrunk compared to that of the existing ITU-based solution. Therefore, the importance of this work lies in not only discovering and confirming the loss gap between the measured air loss and the calculated one in the long-distance propagation case but also providing revision methods to obtain a more accurate prediction for the long-distance air loss at $220 \mathrm{GHz}$. This work paves the way for the development of long-distance terahertz wireless applications

\section{REFERENCES}

[1] T. S. Rappaport, Y. Xing, O. Kanhere, S. Ju, A. Madanayake, S. Mandal, A. Alkhateeb, G. C. Trichopoulos, "Wireless communications and applications above $100 \mathrm{GHz}$ : opportunities and challenges for 6G and beyond," IEEE Access, vol. 7, 2019.

[2] Daniel M. Mittleman, "Perspective: Terahertz science and technology," Journal of Applied Physics, vol. 122, no. 23, 2017.

[3] Y. Xing and T. S. Rappaport, "Terahertz wireless communications: Co-sharing for terrestrial and satellite systems above $100 \mathrm{GHz}$," IEEE Communications Letters, vol. 25, no. 10, pp. 3156-3160, 2021.

[4] J. Ma, R. Shrestha, J. Adelberg, C.-Y. Yeh, Z. Hossain, E. Knightly, J. M. Jornet, and D. M. Mittleman, "Security and eavesdropping in terahertz wireless links," Nature, vol. 563, 2018.

[5] V. Petrov, A. Pyattaev, D. Moltchanov, and Y. Koucheryavy, "Terahertz band communications: Applications, research challenges, and standardization activities," 2016 8th International Congress on Ultra Modern Telecommunications and Control Systems and Workshops (ICUMT), 2016.

[6] J. C. Tucek, M. A. Basten, D. A. Gallagher and K. E. Kreischer, "Operation of a compact $1.03 \mathrm{THz}$ power amplifier," 2016 IEEE International Vacuum Electronics Conference (IVEC), 2016.

[7] M. A. Basten, J. C. Tucek, D. A. Gallagher, K. E. Kreischer and R. Mihailovich, "A $0.85 \mathrm{THz}$ vacuum-based power amplifier," IVEC 2012, 2012.

[8] S. Abadal, S. E. Hosseininejad, A. Cabellos-Aparicio and E. Alarcón, "Graphene-based terahertz antennas for area-constrained applications," 2017 40th International Conference on Telecommunications and Signal Processing (TSP), 2017.

[9] Z.-W. Miao, Z.-C. Hao, G. Q. Luo, L. Gao, J. Wang, X. Wang, and W. Hong, "140 GHz high-gain LTCC-integrated transmit-array antenna using a wideband SIW aperture-coupling phase delay structure," IEEE Transactions on Antennas and Propagation, vol. 66, no. 1, 2018.

[10] J. Ma, R. Shrestha, L. Moeller, and D. M. Mittleman, "Invited Article: Channel performance for indoor and outdoor terahertz wireless links," APL Photonics, vol. 3, no. 5, 2018.

[11] C. Wang, J. Bian, J. Sun, W. Zhang, and M. Zhang, "A survey of 5G channel measurements and models," IEEE Communications Surveys \& Tutorials, vol. 20, no. 4, 2018.

[12] J. F. Federici, J. Ma, and L. Moeller, "Weather impact on outdoor terahertz wireless links," NANOCOM' 15: Proceedings of the Second Annual International Conference on Nanoscale Computing and Communication, 2015.

[13] J. Kokkoniemi, J. Lehtomäki, K. Umebayashi, and M. Juntti, "Frequency and time domain channel models for nanonetworks in Terahertz Band," IEEE Transactions on Antennas and Propagation, vol. 63, no. 2, 2015.

[14] S. Hur, S. Baek, B. Kim, Y. Chang, A. F. Molisch, T. S. Rappaport, K. Haneda, and J. Park, "Proposal on millimeter-wave channel modeling for 
5G cellular system," IEEE Journal of Selected Topics in Signal Processing, vol. 10, no. 3, 2016.

[15] H. Elayan, R. M. Shubair, J. M. Jornet and P. Johari, "Terahertz channel model and link budget analysis for intrabody nanoscale communication," IEEE Transactions on NanoBioscience, vol. 16, no. 6, 2017

[16] M. Shafi, J. Zhang, H. Tataria, A. F. Molisch, S. Sun, T. S. Rappaport, F. Tufvesson, S. Wu, K. Kitao, "Microwave vs. millimeter-wave propagation channels: key differences and impact on $5 \mathrm{G}$ cellular systems," IEEE Communications Magazine, vol. 56, no. 12, 2018.

[17] G. R. MacCartney and T. S. Rappaport, "Rural macrocell path loss models for millimeter wave wireless communications," IEEE Journal on Selected Areas in Communications, vol. 35, no. 7, 2017.

[18] T. S. Rappaport, Y. Xing, G. R. MacCartney, A. F. Molisch, E. Mellios and J. Zhang, "Overview of millimeter wave communications for fifth-generation $(5 \mathrm{G})$ wireless networks - with a focus on propagation models," IEEE Transactions on Antennas and Propagation, vol. 65, no. 12, 2017.

[19] L. Cang, H. Zhao, and G. Zheng, "The impact of atmospheric turbulence on terahertz communication," IEEE Access, vol. 7, 2019.

[20] Y. Golovachev, A. Etinger, G. A. Pinhasi, and Y. Pinhasi, "Propagation properties of sub-millimeter waves in foggy conditions," Journal of Applied Physics, vol. 125, no. 15, 2019.

21] A. Saeed, O. Gurbuz, and M. A. Akkas, "Terahertz communications at various atmospheric altitudes," Physical Communication, vol. 41, 2020.

[22] J. F. Federici, J. Ma, and L. Moeller, "Review of weather impact on outdoor terahertz wireless communication links," Nano Communication Networks, vol. 10, 2016

[23] J. Ma, J. Adelberg, R. Shrestha, L. Moeller and D. M. Mittleman, "The effect of snow on a terahertz wireless data link," Journal of Infrared, Millimeter, and Terahertz Waves, vol. 39, 2018.

[24] Y. Xing and T. S. Rappaport, "Propagation measurements and path loss models for sub-THz in urban microcells," 2021 IEEE International Conference on Communications, June 2021.

[25] E. Moon, T. Jeon and D. R. Grischkowsky, "Long-path THz-TDS atmospheric measurements between buildings," IEEE Transactions on Terahertz Science and Technology, vol. 5, no. 5, 2015.

[26] G. Kim, T. Jeon, and D. Grischkowsky, "910-m propagation of THz ps pulses through the Atmosphere," Opt. Express, vol. 25, no. 21, 2017.

[27] M. K. Samimi and T. S. Rappaport, "3-D millimeter-wave statistical channel model for $5 \mathrm{G}$ wireless system design," IEEE Transactions on Microwave Theory and Techniques, vol. 64, no. 7, 2016.
[28] Y. Xing, T. S. Rappaport, and A. Ghosh, "Millimeter wave and sub-THz indoor radio propagation channel measurements, models, and comparisons in an office environment," IEEE Communications Letters, vol. 25, no. 10, pp. 3151-3155, 2021.

[29] C. Cheng, S. Kim and A. Zajić, "Comparison of path loss models for indoor $30 \mathrm{GHz}, 140 \mathrm{GHz}$, and $300 \mathrm{GHz}$ channels," 2017 11th European Conference on Antennas and Propagation (EUCAP), 2017.

[30] Y. Xing and T. S. Rappaport, "Propagation measurement system and approach at $140 \mathrm{GHz}$-moving to 6G and above $100 \mathrm{GHz}$," 2018 IEEE Global Communications Conference (GLOBECOM), 2018.

[31] Y. Xing, O. Kanhere, S. Ju, and T. S. Rappaport, "Indoor wireless channel properties at millimeter wave and sub-terahertz frequencies," 2019 IEEE Global Communications Conference (GLOBECOM), 2019.

[32] C. Han, A. O. Bicen, and I. F. Akyildiz, "Multi-ray channel modeling and wideband characterization for wireless communications in the terahertz band," IEEE Transactions on Wireless Communications, vol. 14, no. 5, 2015.

[33] 3GPP, "Study on channel model for frequencies from 0.5 to $100 \mathrm{GHz}$," 3rd Generation Partnership Project (3GPP), TR 38.901 V14.0.0, 2017.

[34] ITU-R P.676: https://www.itu.int/rec/R-REC-P.676/en.

[35] S. Sun, T. S. Rappaport, M. Shafi, P. Tang, J. Zhang, P. J. Smith, "Propagation models and performance evaluation for $5 \mathrm{G}$ millimeter-wave bands," IEEE Transactions on Vehicular Technology, vol. 67, no. 9, 2018.

[36] mmMAGIC, "Measurement results and final mmmagic channel models," Tech. Rep. H2020-ICT-671650-mmMAGIC/D2.2, May 2017. [Online]. Available: https://5g-mmmagic.eu/results/

[37] J. Kokkoniemi, J. Lehtomäki, and M. Juntti, "A line-of-sight channel model for the 100-450 gigahertz frequency band," EURASIP Journal on Wireless Communications and Networking, 2021.

[38] I. Llatser, A. Mestres, S. Abadal, E. Alarcón, H. Lee and A. Cabellos-Aparicio, "Time- and frequency-domain analysis of molecular absorption in short-range terahertz communications," IEEE Antennas and Wireless Propagation Letters, vol. 14, 2015.

[39] A. A. Boulogeorgos, E. N. Papasotiriou, J. Kokkoniemi, J. Kokkoniemi, J. Lehtomäki, A. Alexiou, and M. Juntti, "Performance evaluation of THz wireless systems operating in 275-400 GHz," 2018 IEEE 87th Vehicular Technology Conference, 2018.

[40] Q. Jing, D. Liu, and J. Tong, "Study on the scattering effect of terahertz waves in near-surface atmosphere," IEEE Access, vol. 6, 2018.

[41] ITU-R P.835 https://www.itu.int/rec/R-REC-P.835/en 